\title{
ON SOME FUNCTIONALS*
}

\section{BY \\ STANISLAW SAKS $\dagger$}

1. In this paper we intend to give a new proof and various generalizations of the following theorem due to Hahn: $\ddagger$

If $\left\{f_{n}(t)\right\}$ is a sequence of summable functions in the interval $J=(0,1)$ and if $\lim _{n} \int_{E} f_{n}(t) d t$ exists for every measurable set $E \subset J$, then the indefinite integrals $F_{n}(x)=\int_{0}^{x} f_{n}(t) d t$ are equally absolutely continuous in $J$ and therefore converge to an absolutely continuous function.

The proof will be based on a theorem of Baire which has proved useful in many similar cases. Incidentally there will be given a generalization of another theorem concerning sequences of functional transformations and published in a previous paper by the author. 9

2. We shall denote by $R$ the space of measurable characteristic functions in the interval $J=(0,1)$, i.e., functions which almost everywhere assume two values only, 0 and 1 . The distance of two functions $x(t), y(t)$ in $R$ is defined by the formula

$$
d(x, y)=\|x-y\|=\int_{J}|x(t)-y(t)| d t, \quad d(x, 0)=\|x\|=\int_{J}|x(t)| d t .
$$

With this definition of the distance, $R$ is a metric complete space. It is not linear but nevertheless has simple properties which in some cases may replace linearity. We shall state them in the following lemma.

Lemma. (i) If $u$ and $x_{0}$ belong to $R$ and if $\|u\| \leqq r$, then there exist in $R$ elements $u_{1}, u_{2}$ such that

$$
u_{1}=u_{2}+u, d\left(x_{0}, u_{1}\right) \leqq r, d\left(x_{0}, u_{2}\right) \leqq r .
$$

(ii) If $x_{1} \subset R, x_{2} \subset R, d\left(x_{1}, x_{2}\right) \leqq r$, then there exist in $R u_{1}, u_{2}$ such that

$$
\left\|u_{1}\right\| \leqq r,\left\|u_{2}\right\| \leqq r, x_{1}-u_{1} \subset R, x_{2}=x_{1}-u_{1}+u_{2} .
$$

(iii) If $x$ is an element of $R$ and $r>0$, then there exist a finite number of elements $u_{1}, u_{2}, \cdots, u_{n}$ such that $\left\|u_{i}\right\| \leqq r(i=1,2, \cdots, n)$ and

$$
x=u_{1}+u_{2}+\cdots+u_{n} .
$$

* Presented to the Society, October 29, 1932; received by the editors August 24, 1932.

$\dagger$ International Research Fellow.

$\ddagger$ Hahn [1]. Another proof is given in the book of Banach [2, pp. 152-158]. References in brackets refer at the bibliography at the end of this paper.

$\S$ See for instance Banach and Steinhaus [1], Banach [2].

I Saks [1]. 
To prove (i) we simply put

$$
\begin{aligned}
& u_{1}(t)=u(t)+x_{0}(t)[1-u(t)], \\
& u_{2}(t)=x_{0}(t)[1-u(t)] .
\end{aligned}
$$

To prove (ii) we put

$$
\begin{aligned}
& u_{1}(t)=x_{1}(t)\left[1-x_{2}(t)\right], \\
& u_{2}(t)=x_{2}(t)\left[1-x_{1}(t)\right] .
\end{aligned}
$$

To prove (iii) let $n$ be an arbitrary integer $>r^{-1}$. Let $v_{i}(i=1,2, \cdots, n)$ denote the characteristic function of the interval $((i-1) / n, i / n)$. The functions $u_{i}(t)=x(t) v_{i}(t)$ satisfy the condition required by (iii).

The theorem of Hahn will now be stated as follows (in a slightly more general form):

THEOREM 1. If $\left\{f_{n}(t)\right\}$ is a sequence of integrable functions and if the sequence of functionals

$$
F_{n}(x)=\int_{0}^{1} f_{n}(t) x(t) d t, x \subset R,
$$

defined in the space $R$, converges on a set of the second category* $H \subset R$, then the unctionals $F_{n}(x)$ are equally continuous $\dagger_{\text {in }} R$.

We can suppose that the sequence of the functionals $F_{n}(x)$ converges to zero for every $x \subset H$; otherwise we could replace the sequence $\left\{F_{n}(x)\right\}$ by the double sequence $\left\{F_{n}(x)-F_{m}(x)\right\}$. Let $\epsilon$ be an arbitrary positive number. Denote by $H_{n}$ the set of points $x \subset R$ such that $\left|F_{m}(x)\right| \leqq \epsilon / 2$ for every $m \geqq n$. Then

$$
H \subset \sum_{n} H_{n} .
$$

As $H$ is by assumption of the second category there exists a value $n_{0}$ such that $H_{n_{0}}$ is also of the second category. On the other hand, by the continuity of the functionals $F_{n}(x)$, all sets $H_{n}$ are closed; hence $H_{n_{0}}$ contains a sphere, say $\ddagger$ $K=K\left(x_{0} ; r\right)$. Let $u$ be an arbitrary element of $R$ such that $\|u\| \leqq r$. Then, by

* In the sense of Baire. See for instance Hausdorff, Mengenlehre, 1927, pp. 138-145.

$\dagger$ I.e., to every $\epsilon>0$ there corresponds an $\eta>0$ such that $\|x\| \leqq \eta, x \subset R$, implies $\left|F_{n}(x)\right| \leqq \epsilon$ $(n=1,2, \cdots)$. From the equal continuity of the functionals $F_{n}(x)$, it readily follows that under the assumptions of Theorem 1 the sequence $\left\{F_{n}(x)\right\}$ converges everywhere in $R$. For a more general result see below, Theorem 4 (i).

$\ddagger$ In metric spaces, $K\left(x_{0} ; r\right)$ will generally denote the sphere whose center is $x_{0}$ and radius is $r$. 
the preceding lemma, (i), there exist elements $u_{1}, u_{2}$ in $R$ such that $u_{1}=u_{2}+u$, $u_{1} \subset K, u_{2} \subset K$. Therefore for every $n \geqq n_{0}$,

$$
\left|F_{n}(u)\right| \leqq\left|F_{n}\left(u_{1}\right)\right|+\left|F_{n}\left(u_{2}\right)\right| \leqq \epsilon .
$$

Thus the theorem of Hahn is established.

By the same method the following theorem may be proved:

THEOREM 2. If $\left\{f_{n}(t)\right\}$ is a sequence of functions integrable over the interval $J=(0,1)$ and if for all functions $x(t)$ of a set of the second category in the space $R$

then also

$$
\varlimsup_{n}\left|\int_{0}^{1} f_{n}(t) x(t) d t\right|<\infty,
$$

$$
\varlimsup_{n} \int_{0}^{1}\left|f_{n}(t)\right| d t<\infty \text {. }
$$

3. In the sequel we shall consider functional transformations defined either in an arbitrary metric complete linear space or in the space $R$ of characteristic functions (\$2). The values assumed by these transformations will belong to the space $S$ of all measurable functions defined on a measurable set $I$. The distance $d(\xi, \eta)$ of two functions $\xi(t), \eta(t) \subset S$ will be defined by the well known formula of Fréchet

$$
d(\xi, \eta)=\int_{I} \frac{|\xi(t)-\eta(t)|}{1+|\xi(t)-\eta(t)|} d t,
$$

and we put, as usual, $\|\xi\|=d(\xi, 0)$. With this definition of the distance, $\lim _{n} d\left(\xi_{n}, \xi\right)=0\left(\xi_{n}, \xi \subset S\right)$ means that the sequence $\left\{\xi_{n}(t)\right\}$ converges in measure to $\xi(t)$.

Let

$$
\xi=\xi(x, t)=F(x)
$$

be a functional transformation of the kind described above ( $\xi$ is a measurable function, $x$ an element of a metric space* $E, t \subset I$ ). Since $E$ and $S$ are metric spaces it is clear what should be understood by the continuity of the transformation (3.1). This transformation will be called linear if it is continuous and if, for every pair of elements $x, y$ in $E$,

$$
F(x+y)=F(x)+F(y)
$$

provided that the sum $x+y$ is defined and belongs to $E . \dagger$

* For the sake of convenience we shall use the Greek letters $\xi, \eta, \cdots$ to denote the elements of the space $S$, i.e., the measurable functions, and italics, $x, y, \cdots$, for the elements of the space where the transformations (3.1) are defined; $t$ will usually denote points of the set $I$.

$\dagger$ This restriction is necessary in the case of the space $R$ which is not linear. 
Functional transformations

$$
\xi_{n}=\xi_{n}(x, t)=F_{n}(x) \quad(n=1,2, \cdots)
$$

$\left(\xi_{n} \subset S, x \subset E, t \subset I\right)$ will be called equally continuous in the metric space $E$ if to every $\epsilon>0$ there corresponds an $\eta>0$ such that whenever $x^{\prime}, x^{\prime \prime} \subset E$ and $d\left(x^{\prime}, x^{\prime \prime}\right) \leqq \eta$, we have

$$
\left\|\xi_{n}^{\prime}-\xi_{n}^{\prime \prime}\right\| \leqq \epsilon, n=1,2, \cdots, \text { where } \xi^{\prime}=F_{n}\left(x^{\prime}\right), \xi^{\prime \prime}=F_{n}\left(x^{\prime \prime}\right) ;
$$

or, what is equivalent, if to every $\epsilon>0$ there corresponds an $\eta>0$ such that $d\left(x^{\prime}, x^{\prime \prime}\right) \leqq \eta$ implies $\left|\xi_{n}\left(x^{\prime \prime}, t\right)-\xi_{n}\left(x^{\prime}, t\right)\right| \leqq \epsilon$ for all $t \subset I$, with the exception at most of a subset* of $I$ of measure less than $\epsilon$.

If $A$ is a measurable subset of $I$ then the number

$$
d_{A}(\xi, \eta)=\int_{A} \frac{|\xi(t)-\eta(t)|}{1+|\xi(t)-\eta(t)|} d t=\|\xi-\eta\|_{A} \quad(\xi, \eta \subset S)
$$

will be called the distance of $\xi$ and $\eta$ with respect to the set $A$. With this definition it is clear what is meant by the continuity of a transformation (3.1), or the equal continuity and the convergence of a sequence of transformations (3.2), with respect to a measurable set $A \subset I$.

4. LEMMA. If

$$
\xi_{n}=\xi_{n}(x, t)=F_{n}(x) \quad(n=1,2, \cdots)
$$

$\left(x \subset E, t \subset I, \xi_{n} \subset S\right)$ is a sequence of continuous functional transformations in a metric complete space $E$, and if for every element $x$ of a set of the second category $H \subset E$, the inequality

$$
\varlimsup_{n}\left|\xi_{n}(x, t)\right|<\infty
$$

holds on a set of values of $t$ of measure $\alpha>0$, then to every $\epsilon, 0<\epsilon<\alpha$, there correspond a set $A \subset I$ (independent of $x$ ) of measure $\alpha-\epsilon, a$ sphere $K$ in $E$, and a number $M$, such that

$$
\left|\xi_{n}(x, t)\right| \leqq M \quad(n=1,2, \cdots)
$$

for every $x \subset K$ and all $t \subset A$ with the exception at most of a set of values of $t$ of measure zero (which might depend on $x$ ).

Let $\mathfrak{A}$ be a sequence of measurable subsets of the set $I$ everywhere dense in the space of all measurable subsets $\dagger$ of $I$. Let $\left\{A_{m}\right\}$ be a sequence of all sets

* This exceptional subset generally depends on $n$.

$\dagger$ I.e., the set of the characteristic functions of sets of $\mathfrak{A}$ has to be everywhere dense in the space of all measurable characteristic functions defined over $I$ (see §2). 
in $\mathfrak{A}$ whose measure is $\geqq \alpha-\epsilon$. Denote by $H_{m}$ the set of elements $x \subset E$ such that

$$
\left|\xi_{n}(x, t)\right| \leqq m \quad(n=1,2, \cdots)
$$

almost everywhere in $A_{m}$. Then

$$
H \subset H_{1}+H_{2}+\cdots+H_{m}+\cdots \text {. }
$$

$H$ is by assumption of the second category. Hence, there exists a number $M$ such that $H_{M}$ is also of the second category. On the other hand, since the transformations (4.1) are continuous the sets $H_{m}$ are closed, and therefore $H_{M}$ contains a sphere $K$. It is easily seen that the set $A_{M}$, the sphere $K$, and the number $M$ satisfy the required conditions.

ThEOREM 3.* If $\left\{\xi_{n}(x, t)=F_{n}(x)\right\} \quad\left(x \subset E, \xi_{n} \subset S, t \subset I\right)$ is a sequence of linear transformations in a metric complete and linear space $E$, then there exists a set $A \subset I$ such that

$$
\varlimsup_{n}\left|\xi_{n}(x, t)\right|<\infty
$$

at almost every point $t \subset A$ and for every $x \subset E$;

$$
\varlimsup_{n}\left|\xi_{n}(x, t)\right|=\infty
$$

at almost every point $t \subset I-A$, and for every $x \subset E$ with the exception at most of $a$ set of the first category in $E$;

(iii) the transformations $F_{n}(x)$ are equally continuous with respect to the set $A . \dagger$

Let $\alpha_{0}$ be the upper bound of all numbers $\alpha$ such that there exists a set $H(\alpha)$ of the second category in $E$ with the property that for every $x \subset H(\alpha)$

$$
\varlimsup_{n}\left|\xi_{n}(x, t)\right|<\infty
$$

in a subset of $I$ of measure $\alpha$. $\ddagger$ Theorem 3 is trivial when $\alpha_{0}=0$. Hence we may assume $\alpha_{0}>0$. Then, by the preceding lemma, there exists for every $p$ a sphere $K_{p}$, a number $M_{p}$ and a set $A_{p} \subset I$ of measure $\geqq \alpha_{0}-1 / p$ such that for every $x \subset K_{p}$,

$$
\left|\xi_{n}(x, t)\right| \leqq M_{p} \quad(n=1,2, \cdots)
$$

\footnotetext{
* This theorem has been proved in our previous paper (Saks [1]) under the assumption that there exist an everywhere dense set $E_{1}$ in $E$ such that the sequence $\left\{F_{n}(x)\right\}$ converges for every $x \subset E_{1}$.

$\dagger A$ may be empty or coincide with the whole set $I$.

$\ddagger$ This subset in general depends on $x$.
} 
almost everywhere on $A_{p}$. Let

$$
A=\sum_{p} A_{p}
$$

We shall prove that the set $A$ has properties (i), (ii), (iii) above.

First, the inequality (4.2) holds for almost every $t \subset A_{p}$ and every $x \subset K_{p}$, and so, by the linearity of the space $E$, for almost every $t \subset A_{p}$ and every $x \subset E$,

$$
\varlimsup_{n}\left|\xi_{n}(x, t)\right|<\infty \text {. }
$$

Finally, by (4.4) this holds for almost every $t \subset A$ and every $x \subset E$. Hence, property (i) is established.

In order to prove (ii) suppose that there exist a set $H$ of the second category in $E$ such that for every $x \subset H$ the relation (4.2) holds in a subset of $I-A$ of positive measure. Then for every $x \subset H$ this relation would hold in a subset of $I$ of measure $>$ meas $A=\alpha_{0}$, which contradicts the definition of the number $\alpha_{0}$.

Finally, let $r_{p}$ be the radius of the sphere $K_{p}$, and let $x_{0}$ be an arbitrary element in $E$ such that $\left\|x_{0}\right\| \leqq r_{p} /\left(p M_{p}\right)$. It easily follows from (4.3) that

$$
\left|\xi_{n}\left(p M_{p} x_{0}, t\right)\right| \leqq 2 M_{p} \quad(n=1,2, \cdots),
$$

and therefore

$$
\left|\xi_{n}\left(x_{0}, t\right)\right| \leqq \frac{2}{p} \quad(n=1,2, \cdots)
$$

for almost all $t \subset A_{p}$, i.e. everywhere in $A$ with the exception at most of a set of measure $\leqq$ meas $\left(A-A_{p}\right) \leqq 1 / p$. Since $p$ is an arbitrary positive integer, this proves property (iii).

5. The theorem of the preceding section, with the exception of property (iii), may be easily extended to the operations considered in the space $R(\$ 2)$. The proof remains essentially the same, only instead of the linearity of the space $E$ we should use the properties of the space $R$ as stated in the lemma of $\$ 2$. Property (iii) obviously fails for the space $R$. However we have the following theorem:

TheOREM 4. If $\left\{\xi_{n}(x, t)=F_{n}(x)\right\}\left(\xi_{n} \subset S, t \subset I\right)$ is a sequence of linear transformations defined either in a linear metric complete space $E$ or in the space $R$, then there exists $a$ set $B \subset I$ with the following properties:

(i) The functions $F_{n}(x)$ are equally continuous and the sequence $\left\{F_{n}(x)\right\}$ converges with respect to $B$ for every $x$ in $E$ (or in $R$ ). 
(ii) The sequence $\left\{F_{n}(x)\right\}$ diverges with respect to any subset of $I-B$ of positive measure for every $x$ in $E$ (or in $R$ ) with the exception at most of a set of the first category in $E$ (or in $R)$.*

We shall prove this theorem for the space $R$, the proof for the linear spaces being even simpler. We shall use the following lemma which is itself a generalization of the theorem of Hahn of $\$ 2$.

LEMMA. If

$$
\xi_{n}(x, t)=F_{n}(x) \quad(n=1,2, \cdots ; t \subset C \subset I)
$$

(where $C$ is a measurable set) is a sequence of linear transformations in the space $R$ converging with respect to $C$ on a set of the second category $H \subset R$, then $F_{n}(x)$ are equally continuous with respect to $C$ (and therefore $\lim _{n} F_{n}(x)$ is also a linear transformation in $R$ with respect to $C$ ).

The proof runs exactly as that of Hahn's theorem. We can suppose again that the sequence $\left\{\xi_{n}(x, t)=F_{n}(x)\right\}$ converges in $H$ to zero. Then, for every $\epsilon>0$, there exist a number $n_{0}$ and a sphere $K_{0}$ in $R$, of radius $r$, such that

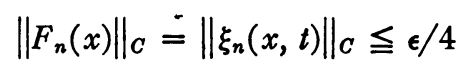

for every $x \subset K_{0}$ and $n \geqq n_{0}$. From (i) and (ii).of the lemma of $\$ 2$ it readily follows that $d_{C}\left(x_{1}, x_{2}\right) \leqq r$ implies $\left\|F_{n}\left(x_{2}\right)-F_{n}\left(x_{1}\right)\right\|_{C} \leqq \epsilon$ for every two points $x_{1}, x_{2}$ in $R$. This proves our lemma.

We now proceed to the proof of Theorem 4 (for the space $R$ ). Denote by $\alpha_{0}$ the upper bound of numbers $\alpha \geqq 0$ with the property that there exists a set $B(\alpha) \subset I$ of measure $\alpha$ such that the given sequence $\left\{F_{n}(x)\right\}$ converges with respect to $B(\alpha)$ for all $x \subset R$. Let $B$ be the sum of all sets $B(\alpha), \alpha \leqq \alpha_{0}$. The set $B$ has both properties (i) and (ii). Indeed, property (i) follows immediately from the definition of $B$ and from the preceding lemma. It remains to establish property (ii). Suppose that for all $x$ of a set of the second category in $R,\left\{F_{n}(x)\right\}$ converges with respect to a subset of $I-B$ of positive measure; this subset depends generally on $x$, but, by the same argument as in the proof of the lemma of $\$ 4$ we can determine a fixed set $C \subset I-B$ of positive measure such that $\left\{F_{n}(x)\right\}$ converges with respect to $C$ for all $x$ of a set of the second category $H \subset R$. There exists a sphere $K_{0}$ in which $H$ is everywhere dense. By the preceding lemma the transformations $F_{n}(x)$ are equally continuous with

* Hence, to any sequence of transformations $\left\{F_{n}(x)\right\}$ in a linear space $E$ there correspond two sets $A$ and $B$ (as defined by Theorems 3 and 4). We have obviously $B \subset A$ (with the exception at most of a set of measure zero). In a special case, if there exists an everywhere dense set $E_{1}$ in $E$ such that the sequence $\left\{F_{n}(x)\right\}$ converges for every $x \subset E_{1}$ (at least with respect to the set $B$ ), it follows easily from the property (iii) of Theorem 3 that $B=A$ (Saks [1], Theorem 6). This obviously is not true for the space $R$. 
respect to $C$. Hence, the sequence $\left\{F_{n}(x)\right\}$ converges everywhere in $K_{0}$. Then, from parts (ii) and (iii) of the lemma of $\$ 2$, it easily follows that this sequence converges (with respect to $C$ ) everywhere in $R$. Thus it converges everywhere in $R$ with respect to the set $B+C \subset I$. Since, by assumption, meas $C>0$ and therefore meas $(B+C)>$ meas $B=\alpha_{0}$, this contradicts the definition of $\alpha_{0}$. Hence our theorem is proved completely.

6. Conclusion. The theorems of $\$ \$ 4$ and 5 may be applied to sequences of transformations of the form $F_{n}(x)=\int_{0}^{1} K_{n}(s, t) x(s) d s=\xi_{n}(x, t)$. For instance* if $\left\{K_{n}(s, t)\right\}$ is a sequence of summable functions (or, more generally, summable with respect to $s$ for almost every value of $t$ ) and if the sequence

$$
\xi_{n}(x, t)=F_{n}(x)=\int_{0}^{1} K_{n}(s, t) x(s) d s \quad(n=1,2, \cdots)
$$

converges for all measurable characteristic functions $x(s)$ (or merely on a set of the second category in $R$ ) then the operations $F_{n}(x)$ are equally continuous. $\dagger$ Therefore, if a sequence of integrals $\int_{P} K_{n}(s, t) d s$ converges in measure in $(0,1)$ for every measurable set $P$ and if there exists a function $f(t)$ such that $\int_{0}^{a} K_{n}(s, t) d s$ converges in measure to $f(t)$ for all $a, 0 \leqq a \leqq 1$, then $\int_{P} K_{n}(s, t) d s$ converges in measure to $f(t)$ for all measurable sets in the interval $(0,1)$.

\section{REFERENCES}

Banach. 1. Sur la convergence presque partout de fonctionnelles linéaires, Bulletin des Sciences Mathématiques, vol. 50 (1926), pp. 27-32, 36-43.

Banach. 2. Teorja operacyj linjowych (in Polish), Warszawa, 1932.

Banach et Steinhaus. 1. Sur le principe de la condensation des singularites, Fundamenta Mathematicae, vol. 9 (1927), pp. 50-61.

Hahn. 1. Über Folgen linearer Operationen, Monatshefte für Mathematik und Physik, vol. 32 (1922), pp. 1-88.

Saks. 1. Sur les fonctionnelles de M. Banach et leur application aux developpements des fonctions, Fundamenta Mathematicae, vol. 10 (1927), pp. 186-196.

* Cf. analogous examples in Banach [1].

$\dagger$ If $K_{n}(s, t)$ reduces to a function of one variable $s$ we find again the theorem of Hahn.

Brown UNIVERSITY, Providence, R. I. 